\title{
Entropy Changes upon Double Layer Charging at a (111)-Textured Au Film in Pure 1-Butyl-1-Methylpyrrolidinium Bis[(trifluoromethyl)sulfonyl]imide lonic Liquid
}

\author{
Jeannette Lindner, ${ }^{\dagger}$ Fabian Weick, ${ }^{\dagger}$ Frank Endres, $^{\ddagger}{ }^{\circledR 0}$ and Rolf Schuster* ${ }^{*}{ }^{\dagger}$ \\ ${ }^{\dagger}$ Institute of Physical Chemistry, Karlsruhe Institute of Technology, 76131 Karlsruhe, Germany \\ ${ }^{\ddagger}$ Institute of Electrochemistry, TU Clausthal, 38678 Clausthal, Germany
}

Supporting Information

\begin{abstract}
We investigated the entropy of the electrical double layer (EDL) formation at a (111)-textured Au film in the ionic liquid 1-butyl-1methylpyrrolidinium bis[(trifluoromethyl)sulfonyl]imide by electrochemical microcalorimetry. We found reversible heat exchange upon EDL charging in the potential range of -0.6 to $1 \mathrm{~V}$ versus $\mathrm{Fc} / \mathrm{Fc}^{+}$. Dependent on the potential, the heat evolution continued for 10 to $200 \mathrm{~ms}$ after the current flow, indicating slow reorganization of the EDL on this time scale. The partial molar entropy of the formation of the EDL $\Delta_{R} S_{\text {form }}$ was derived from the reversibly exchanged heat upon EDL charging. It varied about linearly with the potential from $27 \mathrm{~J} \mathrm{~K}^{-1} \mathrm{~mol}^{-1}$ at $-0.6 \mathrm{~V}$ to $-79 \mathrm{~J} \mathrm{~K}^{-1} \mathrm{~mol}^{-1}$ at $1 \mathrm{~V}$ versus $\mathrm{Fc} / \mathrm{Fc}^{+} . \Delta_{\mathrm{R}} S_{\text {form }}$ crosses zero close to the potential of zero charge of the system. The entropy values are much higher than those arising from purely configurational entropy of a $2 \mathrm{D}$ lattice gas, thus pointing to strong, potential-

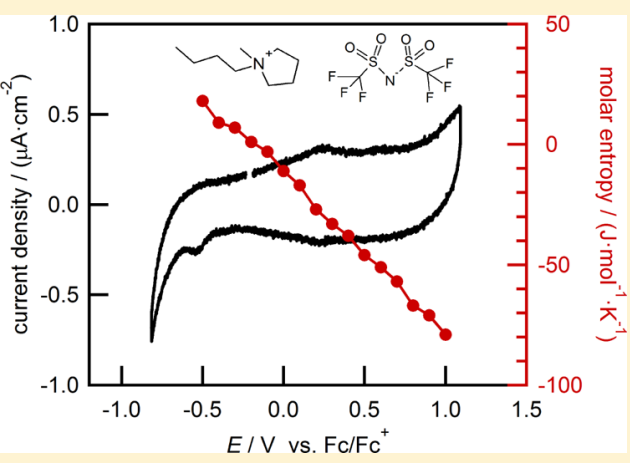
dependent immobilization of the constituents of the EDL, where the immobilization is correlated with the strength of the electric field in the EDL. In addition, from our entropy values, we derive an apparent contradiction with experimentally determined temperature coefficients of the EDL capacitance, which implies a strongly varying activity of the ions participating in charging of the EDL with temperature.
\end{abstract}

\section{INTRODUCTION}

In the last two decades, the growing interest in energy storage has caused tremendous research on water-free electrolytes. One interesting group of candidates is ionic liquids (ILs) because of their large electrochemical window and favorable chemical properties. ${ }^{1-3}$ Although such systems have been extensively investigated theoretically and experimentally (overviews are given, e.g., by Fedorov and Kornyshev, ${ }^{4}$ Hayes et al. ${ }^{5}$ and Perkin ${ }^{6}$ ), fundamental aspects are still not fully clarified. For example, the interface between an IL and a metal surface behaves considerably different from that of a diluted electrolyte. In these "ultra-concentrated electrolytes,"7 mean field descriptions like the Gouy-Chapman model become inapplicable for the description of the electrical double layer (EDL), and the finite size of the ions has to be taken into consideration. ${ }^{8}$ Several theoretical approaches improved the understanding of the experimental observations. 9,10 The potential dependence of the capacitance of the EDL ${ }^{11-13}$ could be rationalized within a mean-field model of a lattice gas, including the finite sizes of the ions. ${ }^{8}$ Overscreening or charge density oscillations in front of the electrode were postulated from continuum theory and simulations ${ }^{8,14}$ and could be correlated to experimental findings from atomic force microscopy (AFM) measurements. ${ }^{10,15,16}$ These showed the formation of ion layers in front of the electrode, extending several layers into the bulk of the IL. Laterally ordered structures of the constituents of the EDL on the electrode surface were observed, for example, by scanning tunneling microscopy (STM), ${ }^{17-22}$ pointing to the importance of longrange surface-specific interactions of the ions. By considering nonelectrostatic interactions, Limmer ${ }^{23}$ found a divergence of the capacitance at $\mathrm{IL} / \mathrm{metal}$ interfaces, signaling a voltagedriven phase transition at the interface.

Experimental information on the structure of the EDL in IL was also obtained, for example, by surface-enhanced infra-red absorption spectroscopy (SEIRAS) $)^{24,25}$ and neutron reflectivity measurements. ${ }^{26}$ On the other hand, information on thermodynamic properties of the EDL is mostly restricted to the measurement of the current-potential or charge-potential relationship of the electrode system, for example, by cyclic voltammetry $(\mathrm{CV})$ or capacitance measurements. ${ }^{27}$ From the thermodynamic point of view, such electrochemical measurements yield the Gibbs free energy of the charging process of the EDL for a certain state of the EDL characterized by the total charge $Q$. In the current contribution, we provide measurements of a complementary thermodynamic quantity, namely, the entropy change during the EDL formation process.

Received: October 21, 2019

Published: December 6, 2019 
The entropy changes during EDL charging should reflect the variations of the ion configuration in the EDL and are therefore expected to be sensitive to the extent of the DL in the bulk of the IL and its composition, to the mobility of the ions as well as to structure formation or phase transitions occurring in the EDL. It should be noted here that recent measurements by the temperature jump method provided information on the potential of maximum entropy of the EDL for [EMMIM][TFSI] (1-ethyl-2,3-dimethyl imidazolium bis[(trifluoromethyl)sulfonyl $]$ imide) on single-crystalline $\mathrm{Pt}$ and $\mathrm{Au}$ electrodes, ${ }^{28,29}$ which, for $\mathrm{Au}$, was found to be close to the potential of zero charge (pzc) and points to a rather disordered ion adlayer in the absence of an electric field. However, no values for the entropy of the EDL formation process were given.

We access the entropy of the EDL formation process by electrochemical microcalorimetry, which allows measuring the heat evolution during the EDL charging process. ${ }^{30,31}$ The reversibly exchanged molar heat at a single electrode, the socalled molar Peltier heat or Peltier coefficient $\Pi$, is given by the sum of the entropy change during the electrochemical process $\Delta_{\mathrm{R}} S$ and a contribution because of ion transport in the bulk of the electrolyte $\Delta S_{\text {Trans }}$, multiplied by the temperature (for details see, e.g., refs 32-34)

$$
\Pi=-T\left(\Delta_{\mathrm{R}} S+\Delta S_{\text {Trans }}\right)
$$

By sign convention, the negative sign is valid for considering the anodic process.

A sufficiently sensitive microcalorimeter was developed in our group. ${ }^{30,35}$ It was already used to successfully measure partial molar reaction entropies in aqueous systems, for example, for $\mathrm{Cu}^{36}$ and $\mathrm{Ag}$ underpotential and bulk deposition $^{31,37}$ on $\mathrm{Au}(111)$ as well as adsorption processes of $\mathrm{SDS}^{38}$ on $\mathrm{Au}(111)$. In this work, we adopt this microcalorimeter to measure the partial molar entropy during double layer charging of $\mathrm{Au}(111)$ in water-free and oxygen-free IL $[\mathrm{BMP}][\mathrm{TFSI}]$ \{1-butyl-1-methylpyrrolidinium bis$[($ trifluoromethyl $)$ sulfonyl $]$ imide $\}$. [BMP $][$ TFSI $]$ is a prototypical IL with high electrochemical stability ${ }^{39,40}$ and has been extensively studied in the literature, for example, via $\mathrm{CV},{ }^{17,19}$ AFM, ${ }^{10,16,41}$ STM, ${ }^{11,19,42}$ electrochemical impedance spectroscopy, ${ }^{11,27,43}$ and neutron reflectometry. ${ }^{26}$ The $[\mathrm{BMP}]^{+}$cation is aliphatic in contrast to aromatic cations like imidazolium, which most probably would react with gold electrodes. ${ }^{44}$ Additionally, cations and anions are similarly sized, ${ }^{45}$ reducing effects of asymmetry on the differential capacitance ${ }^{46}$ and on ion exchange at the surface, as studied via SEIRAS. ${ }^{25}$ From the experimental point of view, it is also important that it is commercially available with very high purity.

\section{EXPERIMENTAL SECTION}

Details on the electrochemical microcalorimeter can be found elsewhere. ${ }^{30,35,47}$ In brief, a $250 \mathrm{~nm}$ thick (111)-textured $\mathrm{Au}$ film on a $50 \mu \mathrm{m}$ thick sapphire sheet is mounted on a thin $\mathrm{LiTaO}_{3}$ pyroelectric sensor. On top of the $\mathrm{Au}$ film, which serves as the working electrode (WE), the electrochemical cell is mounted and sealed with an O-ring. The active area of the WE amounts to $0.2 \mathrm{~cm}^{2}$. The EC cell is completed with two $0.5 \mathrm{~mm}$ thick platinum wires ( $99.999 \%$ purity) serving as reference and counter electrodes. The potential of the Pt quasireference electrode was calibrated versus the ferrocen/ ferrocenium $\left(\mathrm{Fc} / \mathrm{Fc}^{+}\right)$couple in a separate set-up by adding a small grain of ferrocen to the IL and recording the respective
$\mathrm{CV}^{48}$ The Pt quasi-reference showed a potential of $0.06 \pm$ $0.05 \mathrm{~V}$ versus $\mathrm{Fc} / \mathrm{Fc}^{+}$. The error presents the standard deviation of three independent measurements. In the following, all potentials are referenced to the $\mathrm{Fc} / \mathrm{Fc}^{+}$electrode.

$[\mathrm{BMP}][\mathrm{TFSI}]$ was purchased from Merck in UltraPure quality (halides and water $<10 \mathrm{ppm}$, Alkali metal ions $<150$ $\mathrm{ppm}$ ) and stored in a glovebox (MBraun, UniLab) under an $\mathrm{Ar}$ atmosphere $\left(<1 \mathrm{ppm} \mathrm{H}_{2} \mathrm{O},<2 \mathrm{ppm} \mathrm{O}_{2}\right)$. For all measurements, the IL was used as received without further cleaning. Prior to use, platinum counter and reference electrodes, cell and O-ring were boiled twice in Caroic acid, repeatedly rinsed with ultrapure water (Arium, Sartorius AG) and dried at 100 ${ }^{\circ} \mathrm{C}$ for at least 2 days. The WE was stored for at least one day at $600{ }^{\circ} \mathrm{C}$ in a quartz tube furnace and was freshly flame annealed to achieve a well-ordered (111)-textured film, ${ }^{49}$ before installing it in the cell. The fully assembled microcalorimeter was attached to the glovebox and filled with roughly $300 \mu \mathrm{L}$ IL under an inert atmosphere. To avoid contamination of the $\mathrm{Au}$ surface, assembly and filling were completed within less than $15 \mathrm{~min}$ after the flame annealing of the sample. The calorimetric measurements were then conducted under inert gas atmosphere. Calibration of the calorimeter was performed after the measurements in IL by exchanging the electrolyte for an aqueous $0.1 \mathrm{M}\left[\mathrm{Fe}(\mathrm{CN})_{6}\right]^{3-} /$ $0.1 \mathrm{M}\left[\mathrm{Fe}(\mathrm{CN})_{6}\right]^{4-}$ solution and measuring the Peltier heat for the electron transfer reaction of this redox couple (for details see ref 35 ). In the literature, a Peltier heat of $\Pi=-45.1 \mathrm{~kJ} / \mathrm{mol}$ is given for this reaction. ${ }^{50}$

\section{RESULTS}

3.1. Cyclic Voltammogram. Figure 1 shows two successive scans of a cyclic voltammogram of $\mathrm{Au}$ in

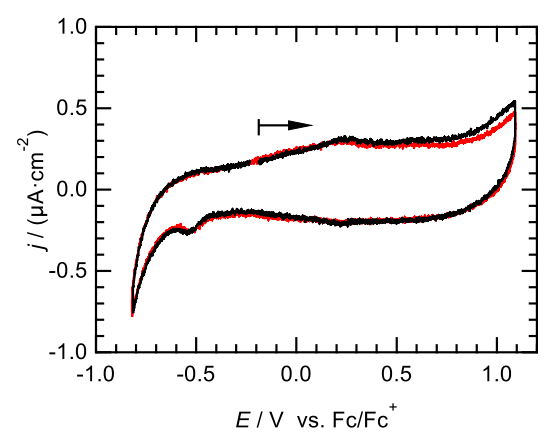

Figure 1. First (black trace) and second (red trace) cycle of a cyclic voltammogram of $\mathrm{Au}(111)$ in "pure" [BMP] [TFSI] (i.e., $<10 \mathrm{ppm}$ water) under an inert gas atmosphere. Scan speed: $10 \mathrm{mV} / \mathrm{s}$.

$[\mathrm{BMP}][\mathrm{TFSI}]$, recorded in situ in the calorimeter cell with a scan rate of $10 \mathrm{mV} / \mathrm{s}$. The current density remained well within $\pm 1 \mu \mathrm{A} / \mathrm{cm}^{2}$, and the total charge which flowed during the complete anodic or cathodic cycle amounts to about \pm 50 $\mu \mathrm{C} / \mathrm{cm}^{2}$. This value is well below that of a monolayer of elementary charges referenced to the number of gold atoms in the topmost surface layer (ca. $\left.220 \mu \mathrm{C} / \mathrm{cm}^{2}\right) .{ }^{51}$ The $\mathrm{CV}$ is essentially flat. The very small peak pair at $-0.2 \mathrm{~V}$ is attributed to traces of water because its height increases when water is added to the IL (see Supporting Information, Figure S1). The small cathodic peak at $-0.55 \mathrm{~V}$ was always present in "ultrapure" [BMP][TFSI]. From Video STM-studies by Wen and Magnussen, ${ }^{19}$ we do not expect structure formation of the IL at the interface at this potential. Thus, we rather attribute 
this current wave to adsorption of inevitable traces of impurities from the production process of the IL. A comparison to a $\mathrm{CV}$ by Zhong et $\mathrm{al}^{41}$ for the same system, [BMP][TFSI] on $\mathrm{Au}(111)$, showed similar potential window and current densities. However, it was not possible to exactly correlate their SCE potential scale to our $\mathrm{Fc} / \mathrm{Fc}^{+}$scale. The two successive scans of the CV in Figure 1 nicely lay on the top of each other, demonstrating the reversibility of the electrochemical processes in the investigated potential interval. For comparison, Figure S3 in the Supporting Information shows the effect of widening the potential window to $-2.5 \mathrm{~V}$ on the cathodic side. The two cathodic peaks at $E \approx-1.0$ and $-1.5 \mathrm{~V}$ were attributed by STM to the formation of the cation monolayer ${ }^{19}$ and by mass spectrometry studies to the decomposition of the IL. ${ }^{40}$ During the subsequent positive cycle, an anodic peak at $E \approx-0.08 \mathrm{~V}$ appears, correlated with the decomposition of the IL at potentials below ca. $-1.0 \mathrm{~V}$ (see Supporting Information, Figure S2). Thus, to avoid such irreversible processes during microcalorimetric measurements, we restricted the investigated potential interval to $-0.6 \mathrm{~V}<E<$ $+1.0 \mathrm{~V}$.

3.2. Microcalorimetry and Peltier Heats. The result of a typical microcalorimetric experiment for the charging of the EDL by $-0.1 \mathrm{~V}$ starting from a rest potential of $E=+0.7 \mathrm{~V}$ is shown in Figure 2. At $t=10 \mathrm{~ms}$, a potential pulse of $-0.1 \mathrm{~V}$

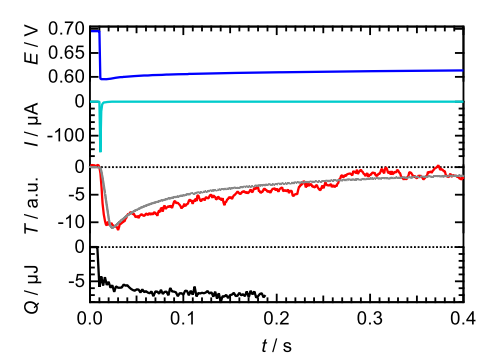

Figure 2. Potential $E$ (dark blue trace), current $I$ (light blue trace), and temperature $T$ transients (red trace), following a $-0.1 \mathrm{~V}$ potential pulse starting from $E=+0.7 \mathrm{~V}$ vs $\mathrm{Fc} / \mathrm{Fc}^{+}$. The thermal response of the electrode-sensor assembly determined by irradiation with a $10 \mathrm{~ms}$ laser pulse is included as gray line. The amount of the exchanged heat $Q$ dependent on the time is given as black trace.

was applied to the WE (dark blue trace). This caused a negative charging current spike (light blue trace), which leveled off a few milliseconds after the potential step. At $t=20$ $\mathrm{ms}$, the cell was switched to open circuit conditions, that is, the outer cell circuit is interrupted, and the potential relaxes by about $15 \mathrm{mV}$ on a time scale of the next $200 \mathrm{~ms}$. The red trace shows the evolution of the electrode temperature during the charging process. In parallel with the current spike, the temperature of the electrode steeply decreased and reached its minimum roughly $10 \mathrm{~ms}$ after the potential step was applied. This indicates that heat was consumed during the charging processes in the EDL, which led to cooling of the electrode system. After charging, the current ceased and the temperature increased on a timescale of several $100 \mathrm{~ms}$ toward the temperature before the potential pulse was applied. For comparison, the expected thermal response following a 10 $\mathrm{ms}$ long square-shaped negative heat pulse is included in Figure 2 (gray trace). It is calculated from measuring the thermal response function of the electrode-sensor assembly by irradiation with laser pulses. ${ }^{37}$ It should be noted here that in Figure 2 the absolute temperature decrease is of the order of a few $10 \mu \mathrm{K}$, which is much lower than those observed in previous studies of aqueous systems, primarily because of the very low charging current of the EDL in ILs. From the temperature transients of Figure 2, it is noticeable that up to about $200 \mathrm{~ms}$, the measured thermal equilibration because of heat uptake from the surrounding (red line) is slower than the temperature increase, expected for a $10 \mathrm{~ms}$ heat pulse (gray line). This can be seen more clearly by reconstruction of the heat flux with the thermal response function of the cell, following the procedure given in ref 37 . The total heat uptake versus time, resulting from the integration of the heat flux, is also included in Figure 2, lowest panel (black trace). This detailed analysis reveals that the electrode significantly continued to cool for about $50 \mathrm{~ms}$, albeit no charging current flowed in the outer cell circuit. This suggests that after the potential step, relaxation processes of the EDL continued for several $10 \mathrm{~ms}$. Similarly, slow relaxation processes were also found, for example, via impedance measurements by Roling and co-workers. ${ }^{11,52,53}$ These authors propose that EDL charging, that is, capacitive processes in the double layer, should be finished within $t<200 \mathrm{~ms}{ }^{54}$ In our measurements, the time scale of the relaxation processes varied between several $10 \mathrm{~ms}$ and about $200 \mathrm{~ms}$, dependent on both, the polarity of the potential pulse and the rest potential before the potential pulse. We cannot fully exclude that heat evolution continues after $200 \mathrm{~ms}$. However, thermal drift of the sensor signal will become increasingly deteriorating for longer timescales. From carefully checking the influence of the upper integration time boundary on the total heat values, we found that $200 \mathrm{~ms}$ is a good compromise between increasing error because of thermal drift and errors because of neglected heat evolution. In the following, we thus calculate the total heat of the EDL processes by integration of the heat flux up to 200 ms. The error because of the integration time boundary is included in the error bars of the following results.

The negative sign of the temperature change in Figure 2 already signals that the heat evolution is dominated by reversible heat effects because irreversible heat contributions will always lead to heating of the system. In order to quantitatively determine the reversible contribution to the heat effects, we conducted pulse experiments as in Figure 2 with different potential-step amplitudes and polarities. We calculated the molar heat of the EDL charging process by dividing the heat by the moles of elementary charges, which flowed in the outer cell circuit during the potential step. The results for the molar heat values of such a series of experiments at a rest potential of $E=+0.7 \mathrm{~V}$ are shown in Figure 3. Extrapolation of the measured molar heats to zero overpotential gives the reversible molar heat of the EDL charging process at $E=+0.7$ $\mathrm{V}$, that is, the corresponding molar Peltier heat. We repeated such series of pulse experiments for different rest potentials by stepwise varying the rest potential by $0.1 \mathrm{~V}$ in the potential interval between -0.6 and $+1.0 \mathrm{~V}$. After stepping the starting potential to a new value, the system was given $1 \mathrm{~min}$ to equilibrate to account for eventual slow relaxation processes of the EDL, ${ }^{53,54}$ before potential pulses with different amplitudes were applied. Figure 4 presents the results for four series of starting potential variations obtained with the same cell assembly (orange and red). The direction of the rest potential variation is indicated by the orientation of the triangles. The estimated error of the fitting procedure of the Peltier heat is about $\pm 3 \mathrm{~kJ} / \mathrm{mol}$. 


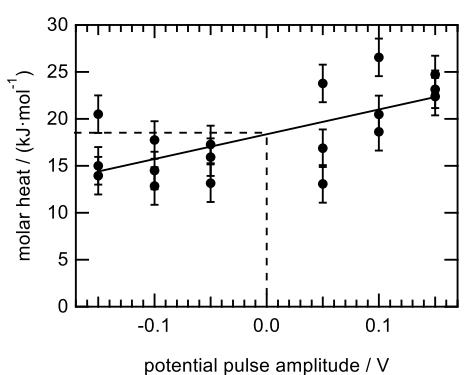

Figure 3. Molar heat, that is, the heat exchanged during $200 \mathrm{~ms}$ after the beginning of the potential pulse, normalized by the amount of charge, which flowed in the cell circuit, is given for a series of potential pulse experiments with different pulse amplitudes. The rest potential before the pulses were applied was $E=+0.7 \mathrm{~V}$ vs $\mathrm{Fc} / \mathrm{Fc}^{+}$.

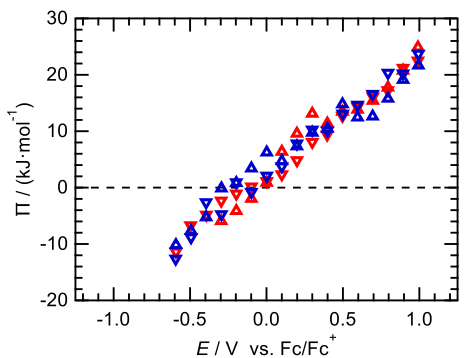

Figure 4. Peltier heat $\Pi$, that is, the reversible molar heat, is shown as a function of the rest potential before the potential pulse. Data for four series of experiments are given. The direction of the variation of the rest potential during the individual series is indicated by the orientation of the triangles (positive variations: triangles pointing up).

By convention, positive Peltier heat corresponds to warming during the anodic process and cooling during the cathodic process. In Figure 4, the molar Peltier heat varies almost linearly with the potential of the EDL, changing its sign at about $-0.2 \mathrm{~V}$ and spanning a range from approximately -13 to $+25 \mathrm{~kJ} / \mathrm{mol}$ upon potential variation between -0.6 to $+1.0 \mathrm{~V}$. There is no evident influence of the direction of the potential variation on the measured molar Peltier heats. Although the underlying heat values were tiny, the same trend of increasing Peltier heat with increasing potential is clearly observable in all series of measurements.

3.3. Molar Entropy of EDL Formation $\Delta_{R} S_{\text {form }}$. As shown in eq 1 , the molar Peltier heat $\Pi$ is determined by the reaction entropy $\Delta_{R} S$ of the electrochemical process and a contribution $\Delta S_{\text {Trans }}$ because of transport of ions in the bulk of the electrolyte and electrons in the electrode. For further discussion, the importance of $\Delta S_{\text {Trans }}$ should be clarified. Its magnitude depends on the Hittorf transference numbers and the so-called Eastman entropies of transport of the ions in solution and electrons in the electrode. ${ }^{32-34}$ Because the solution composition does not change in our experiments, the contribution from transport is constant. Definite numbers for the Eastman entropies of the IL ions do not exist. For hightemperature molten salts of monovalent ions, values between 8 and $20 \mathrm{~J} \mathrm{~K}^{-1} \mathrm{~mol}^{-1}$ were reported. Because the ions of ILs are typically much larger than those of high-temperature molten salts, their Eastman entropies are expected to be even smaller. ${ }^{55}$ This is supported by applying an approximation by Agar. $^{32}$ Using his formula and employing the temperature dependence of the dielectric constant as measured by dielectric relaxation spectroscopy ${ }^{56}$ for [BMIM] [TFSI] and the diffusion coefficients $^{57}$ of $[\mathrm{BMP}]^{+}$and $[\mathrm{TFSI}]^{-}$, we estimated that the Eastman entropies of transport for ions in the IL will be smaller than about $1 \mathrm{~J} \mathrm{~K}^{-1} \mathrm{~mol}^{-1}$. ${ }^{47}$ Considering that both ions participate about equally to the cell current but move into opposite directions, their contributions to $\Delta S_{\text {Trans }}$ partially cancel so that the net contribution to the entropy changes from transport is expected to be well below $1 \mathrm{~J} \mathrm{~K}^{-1} \mathrm{~mol}^{-1}$, which corresponds to a contribution to the Peltier heat of less than $0.3 \mathrm{~kJ} / \mathrm{mol}$ at room temperature, much less than the error bars of our measurement. Thus, $\Delta S_{\text {Trans }}$ can be safely neglected in our study, and the Peltier heat directly reflects the entropy changes of the electrochemical process.

In the absence of Faradaic processes, which is reasonable to assume from the $\mathrm{CV}$ shown in Figure 1, the relevant electrochemical processes in the potential window between -0.6 and $+1.0 \mathrm{~V}$ correspond to capacitive charging of the EDL at the $\mathrm{Au}$ electrode. The entropy changes of the electrochemical processes therefore correspond to the changes of the entropy of the EDL during the charging process, including all side processes like reordering of the interface, reorientation of IL molecules, and so forth. For this entropy, Harrison et al. coined the expression "entropy of formation of the double layer", $S_{\text {form. }}{ }^{58}$ In their words, this entropy corresponds to the difference in entropy of the constituents of the double layer to their values when they are part of the bulk of the electrolyte (see also discussion in ref 59). In our experiment, we directly measure the variation of $S_{\text {form }}$ with the charge $Q$ which flowed in the cell circuit, that is, we measure $\partial S_{\text {form }} / \partial Q . \partial S_{\text {form }} / \partial Q$ is referenced to moles of elementary charges or "equivalents" and thus corresponds to the partial molar entropy of formation of the EDL, $\Delta_{\mathrm{R}} S_{\text {form }}$. In Figure 5, we show an average for this

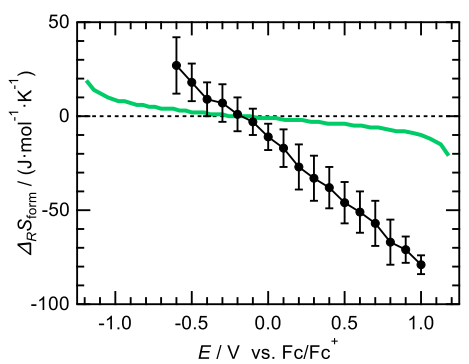

Figure 5. Partial molar entropy for the EDL formation, $\Delta_{\mathrm{R}} S_{\text {form }}$, obtained by averaging eight series of experiments (black dots). Error bars correspond to the standard deviation. The green line indicates the partial molar entropy expected from the configurational entropy of an ideal $2 \mathrm{D}$ lattice gas.

quantity extracted from eight series of experiments and three different electrolyte cell assemblies (black circles). Error bars represent the standard deviation from the average. It should be noted that the error bars in Figure 5 are somewhat larger than those we usually found for aqueous systems (e.g., refs 31 and 36). This is because of the small charging currents and long time scales for EDL charging in ILs. In addition, variations of the potential of the $\mathrm{Pt}$ pseudo-reference electrode (see Experimental Section) may add to the error.

The partial molar entropy of EDL formation is linearly varying with potential and crosses zero at about $-0.2 \mathrm{~V}$. This should be compared with impedance measurements of Lockett et al. $^{27}$ They found a capacitance minimum that they interpreted as pzc at $E_{\mathrm{pzc}}=-0.45 \mathrm{~V}$ versus $\mathrm{Ag} / \mathrm{Ag}^{+}$, which corresponds to $E \approx-0.1 \mathrm{~V}$ versus $\mathrm{Fc} / \mathrm{Fc}^{+}$. Within the scope of 
uncertainty of our measurements, this coincides reasonably well with the zero crossing of $\Delta_{\mathrm{R}} S_{\text {form }}$. It is noteworthy that Zhong et al. determined a pzc of $-0.11 \mathrm{~V}$ versus SCE in $[\mathrm{BMP}][\mathrm{TFSI}] / \mathrm{Au}(111)$ with a water content of $35 \mathrm{ppm}$ via immersion experiments. ${ }^{41}$ However, the corresponding value on our potential scale is unknown, and a comparison of the first cathodic current waves in the CVs is very difficult.

\section{DISCUSSION}

4.1. Variation of $\Delta_{R} S_{\text {form }}$ with Potential. Together with the negative slope, the zero crossing of $\Delta_{\mathrm{R}} S_{\text {form }}$ implies a maximum of the total entropy of formation of the EDL, $S_{\text {form }}$ close to the pzc. This is in line with temperature jump measurements of Sebastián et al. of Au surfaces in [EMMIM][TFSI]. They measured the potential transient following shortpulse laser heating of the surface. Positive signals indicate positive temperature coefficients of the potential and thus negative partial molar entropy of the double layer formation process and vice versa. The potential transients changed their sign from negative to positive close to the pzc upon increasing the potential, signaling a maximum of the entropy close to the pzc, in full accordance with our measurements. Unfortunately, in these experiments, no absolute entropy values for the double layer formation process could be determined. In fact, we are not aware of other reports on the entropy of EDL in IL. However, there is a direct relation between the partial molar entropy of EDL formation and another thermodynamic quantity of the EDL, the temperature derivative of the capacitance, as originally derived by Harrison et al. ${ }^{58}$ (see also ref 60 )

$$
\Delta_{\mathrm{R}} S_{\text {form }}=\left(\frac{\partial S_{\text {form }}}{\partial Q}\right)_{a_{i}, T, p}=-\left(\frac{\partial \Phi}{\partial T}\right)_{a_{i}, Q, p}
$$

where $\Phi=\left(E-E_{\mathrm{pzc}}\right)$ is the single-electrode potential referenced to the pzc, $a_{\mathrm{i}}$ is the activity of component $i$ in the bulk of the electrolyte, and $p$ is the pressure. For purely capacitive processes, the potential with respect to the pzc can be expressed via the (differential) capacitance of the EDL $C=$ $\partial Q / \partial \Phi$ by $\Phi=\int_{0}^{Q} \frac{1}{C} \mathrm{~d} Q^{\prime}$, where $Q$ is the charge at the metal surface at the respective potential $\Phi$. This yields

$$
\left(\frac{\partial S_{\text {form }}}{\partial Q}\right)_{a_{i}, T, p}=-\frac{\partial}{\partial T}\left(\int_{0}^{Q} \frac{1}{C} \mathrm{~d} Q^{\prime}\right)_{a_{i}, Q, p}
$$

For the slope of the $\Delta_{\mathrm{R}} S_{\text {form }}$ versus potential curve, we obtain

$$
\begin{gathered}
\frac{\partial \Delta_{\mathrm{R}} S_{\text {form }}}{\partial \Phi}=\left(\frac{\partial^{2} S_{\text {form }}}{\partial Q \partial \Phi}\right)_{a_{i}, T, p}=\left(C \frac{\partial^{2} S_{\text {form }}}{\partial Q^{2}}\right)_{a_{i}, T, p} \\
=-C\left(\frac{\partial}{\partial T} \frac{1}{C}\right)_{a_{i}, Q, p}=\frac{1}{C} \frac{\partial C}{\partial T}
\end{gathered}
$$

which corresponds to the temperature coefficient of the capacitance. Thus, the negative slope of the partial molar entropy of EDL formation with potential in Figure 5 directly indicates a negative temperature coefficient of the capacitance. Because the capacitance is about constant in the investigated potential interval $\left(C \approx 8.5 \mu \mathrm{F} / \mathrm{cm}^{2}\right.$, which is in accordance with the values given by ref 27), from Figure 5, we readily estimate a normalized temperature coefficient of the capacitance of approximately $-10^{-3} 1 / \mathrm{K}$.

Chen et al. recently presented theoretical predictions and simulations on the temperature dependence of the EDL capacitance derived from the Goodwin-Kornyshev mean field description of a 3D lattice gas model. ${ }^{7}$ By inclusion of ion pairing and formation of neutral aggregates, which are termed "spectating ions" and which do essentially not take part in the charging of the EDL, this model can reproduce the general "camel-like" shape of the capacitance versus potential curve. It shows a transition from a "camel-like" to a bell-shaped capacitance curve with increasing temperature, which was attributed to the amount of "participating ions". The latter increases with increasing temperature by thermal dissociation of "spectating" agglomerates. For the rescaled Debye capacitance, which corresponds to the capacitance at the pzc, Chen et al. found negative temperature coefficients of some $-10^{-3} 1 / \mathrm{K}$ at high temperatures or small amounts of "spectating ions". This value comes close to those calculated from our entropy data. However, at high fractions of "spectating ions", that is, at high energy differences between "participating" and "spectating ions" or low temperatures, thermal excitation of "spectating ions" to "participating ions" has to be considered, which results in a rather strongly positive temperature coefficient of the EDL capacitance. Positive temperature coefficients with magnitudes up to about $10^{-2}$ $1 / \mathrm{K}$ are also reported from several experimental studies, for example, for $[\mathrm{BMIM}]\left[\mathrm{PF}_{6}\right]$ on glassy carbon by Silva et al. ${ }^{61}$ and for [BMIM] $[\mathrm{Cl}]$ on glassy carbon by Lockett et al. ${ }^{62}$ Drüschler et al. ${ }^{52}$ found similar temperature coefficients for $[\mathrm{BMP}][\mathrm{FAP}]$ on $\mathrm{Au}(111)$, when measuring the capacitance on slow timescales $(<200 \mathrm{~ms})$, which is comparable to the time scale of our experiments.

In conclusion, temperature coefficients measured directly or calculated for EDL models show the opposite sign and are almost an order of magnitude larger than the ones expected from our entropy values on the basis of thermodynamic considerations. How can this apparent discrepancy be resolved? The key may be found in the constraints of eq 2 . This equation is only valid, if the activities of the components are kept constant. Following the model of Chen et al., ${ }^{7}$ this may not be the case. The thermal excitation of "spectating ions" to "participating ones" changes the activity of the components, which take part in EDL charging. In fact, if the influence of thermal excitation of "spectating ions" becomes small, that is, at high temperatures or small interactions, the theoretically expected temperature coefficient becomes comparable to the one derived from our entropy data.

4.2. Origin of $\Delta_{R} S_{\text {form }}$. As discussed above, the zero crossing of $\Delta_{\mathrm{R}} S_{\text {form }}$ implies a maximum of the total entropy of EDL formation close to the pzc, where equal amounts of both ions are present in the EDL. This suggests a comparison with the entropy of mixing, that is, the configurational entropy of a simple ideal 2D lattice gas. ${ }^{63}$ In its simplest form, an ideal twocomponent lattice gas shows a maximum of the total entropy at a mole fraction of $x=0.5$ and zero total entropy of mixing at $x$ $=0$ and $x=1$, where $x=0$ corresponds to the full cation monolayer and $x=1$ to the full anion monolayer; $x=0.5$ corresponds to the pzc, where cations and anions are present in equal amounts. For comparison in Figure 5, we included the partial molar entropy of such a 2D lattice gas model (dashed green line), where the mole fraction was adapted to the potential scale, that is a full cation monolayer at $E=-1.2 \mathrm{~V}^{19}$ 
and a full anion monolayer at $E=+1.2 \mathrm{~V}$ (using the diameter ratio $^{18}$ of the anions and cations at the surface of $\left.0.6: 1\right)$. The partial molar entropy is referenced to moles of elementary charges flowing in the outer circuit. Although at intermediate potentials, the partial molar entropy of the lattice gas varies about linearly with potential, and it remains about an order of magnitude smaller than the experimentally observed one. Therefore, the purely configurational entropy of the ions in a single 2D adlayer cannot explain the experimental data. Including particle interactions, for example, by the quasichemical approximation, ${ }^{64}$ would not substantially alter this conclusion. Moreover, several theoretical groups, for example, Kornyshev and Qiao ${ }^{65}$ proposed a rather $3 \mathrm{D}$ instead of $2 \mathrm{D}$ restructuring of the EDL which is in line with the formation of multilayers as found for this system by AFM. ${ }^{66}$ However, considering the configurational entropy of several ordered layers does also not seem to account for the experimentally determined partial molar entropy of EDL formation.

Our entropy data thus indicate that the entropy of the formation of the EDL is determined by entropic contributions, much stronger than purely configurational ones. So far, we neglected possible constraints on the ion mobility when they enter the EDL from the bulk of solution. Upon complete immobilization of the ions in the bulk liquid, that is upon freezing, the partial molar entropy change reaches $-83 \mathrm{~J} \mathrm{~K}^{-1}$ $\mathrm{mol}^{-1}$, as determined for freezing of [BMP][TFSI] via differential scanning calorimetry by Tokuda et al. ${ }^{57}$ This value is indeed close to the experimentally observed partial molar entropy for the EDL formation at $E=+1.0 \mathrm{~V}$ (see Figure 5 ), where an almost complete anion layer is expected for the EDL. This implies about complete ion immobilization, when additional anions are incorporated into an almost complete anion layer. At negative potentials, the span of our data does not reach the full monolayer cation coverage, but extrapolation of the data in Figure 5 to a full cation coverage at about $-1.2 \mathrm{~V}$ yields again absolute values for the partial molar entropy of about $+80 \mathrm{~J} \mathrm{~K}^{-1} \mathrm{~mol}^{-1}$. Note that cations are expelled from a cation-rich layer for the anodic process direction, which is the process direction underlying our partial molar entropy data in Figure 5. Thus, our data also imply an about complete immobilization of the cations in the complete cation layer at strongly negative potentials. For intermediate potentials, the partial molar entropy approaches zero, indicating that the immobilization of the ions decreases upon coming closer to the pzc. At the pzc, where both cation and anions are present in the EDL with equal amounts, $\Delta_{\mathrm{R}} S_{\text {form }}$ becomes zero, signaling that the ions enter the EDL without significant changes of their mobility. When the EDL becomes anion-rich at more positive potentials, the immobilization increases again, and complete immobilization is reached at the full anion layer. It therefore seems that the degree of immobilization is correlated with the strength of the electric field in the EDL. Our results are therefore in line with electrostriction acting on the ions in the EDL, possibly leading to a compaction of the ion layers, as recently found by Georgi et al. ${ }^{67}$ by a Monte Carlo simulation of an IL with anisotropic ions.

\section{CONCLUSIONS}

The double layer charging of (111)-textured Au films in the IL [BMP][TFSI] was investigated by electrochemical microcalorimetry. We measured the heat changes upon double layer charging by short potential pulses. The observed heat changes were essentially completed after about 10-200 ms, dependent on the rest potential, which is in line with impedance spectroscopy and other data signaling a rather slow charging process of the EDL in IL and which was attributed to strong rearrangement of the ions in the EDL. The heat changes were reversible with the pulse polarity. Thus our measurements allowed the determination of the partial molar entropy for the EDL formation process $\Delta_{\mathrm{R}} S_{\text {form }}$ in ILs. The molar entropy displays a strong, nearly linear decrease with increasing potential from $27 \mathrm{~J} \mathrm{~K}^{-1} \mathrm{~mol}^{-1}$ at $-0.6 \mathrm{~V}$ to $-79 \mathrm{~J} \mathrm{~K}^{-1}$ $\mathrm{mol}^{-1}$ at $1 \mathrm{~V}$ versus $\mathrm{Fc} / \mathrm{Fc}^{+}$. Zero crossing occurs at $E=-0.2$ $\mathrm{V}$, which is comparable to the pzc given in the literature. The zero crossing of the partial molar entropy indicates a maximum of the corresponding entropy of formation of the EDL close to the pzc, which is in line with recent temperature jump experiments. From the slope of the $\Delta_{R} S_{\text {form }}$ curve with potential, we estimated a temperature coefficient of the capacitance of about $-10^{-3} 1 / \mathrm{K}$ by use of a thermodynamic relation. This value contrasts those found experimentally for the EDL in several ILs, which were positive and larger by about one order of magnitude. This apparent discrepancy points to other contributions to the temperature coefficient of the capacitance in addition to entropic ones, for example, originating from changes in the activity of the ions participating in the EDL formation.

The determined values for $\Delta_{\mathrm{R}} S_{\text {form }}$ strongly exceed those expected for the purely configurational entropy of a $2 \mathrm{D}$ ion adlayer and thus point to strong restrictions of the mobility of the ions in the EDL compared to that in the bulk of the IL. From the potential variations of $\Delta_{R} S_{\text {form }}$, we conclude that the immobilization of the ions increases with increasing field strength of the electric field in the EDL, signaling strong electrostriction effects in the EDL.

\section{ASSOCIATED CONTENT}

\section{S Supporting Information}

The Supporting Information is available free of charge at https://pubs.acs.org/doi/10.1021/acs.jpcc.9b09871.

Comparison of 'water-free' and moist [BMP][TFSI] and cyclic voltammograms of 'pure' [BMP][TFSI] for medium to large potential windows (PDF)

\section{AUTHOR INFORMATION}

\section{Corresponding Author}

*E-mail: Rolf.Schuster@kit.edu.

ORCID

Frank Endres: 0000-0002-5719-7241

Rolf Schuster: 0000-0003-3136-6403

Notes

The authors declare no competing financial interest.

\section{ACKNOWLEDGMENTS}

We thank Sandra Schmidt (KIT Karlsruhe) for experimental assistance and Natalia Borisenko (TU Clausthal) for the introduction to handling ILs. This work contributes to the research performed at the "Cluster of Excellence" POLiS of the Deutsche Forschungsgemeinschaft and at CELEST (Center for Electrochemical Energy Storage Ulm-Karlsruhe).

\section{REFERENCES}

(1) MacFarlane, D. R.; Tachikawa, N.; Forsyth, M.; Pringle, J. M.; Howlett, P. C.; Elliott, G. D.; Davis, J. H.; Watanabe, M.; Simon, P.; 
Angell, C. A. Energy applications of ionic liquids. Energy Environ. Sci. 2014, 7, 232-250.

(2) Armand, M.; Endres, F.; MacFarlane, D. R.; Ohno, H.; Scrosati, B. Ionic-liquid materials for the electrochemical challenges of the future. Nat. Mater. 2009, 8, 621-629.

(3) Wishart, J. F. Energy applications of ionic liquids. Energy Environ. Sci. 2009, 2, 956.

(4) Fedorov, M. V.; Kornyshev, A. A. Ionic liquids at electrified interfaces. Chem. Rev. 2014, 114, 2978-3036.

(5) Hayes, R.; Warr, G. G.; Atkin, R. Structure and nanostructure in ionic liquids. Chem. Rev. 2015, 115, 6357-6426.

(6) Perkin, S. Ionic liquids in confined geometries. Phys. Chem. Chem. Phys. 2012, 14, 5052-5062.

(7) Chen, M.; Goodwin, Z. A. H.; Feng, G.; Kornyshev, A. A. On the temperature dependence of the double layer capacitance of ionic liquids. J. Electroanal. Chem. 2018, 819, 347-358.

(8) Kornyshev, A. A. Double-layer in ionic liquids: paradigm change? J. Phys. Chem. B 2007, 111, 5545-5557.

(9) Smith, A. M.; Lee, A. A.; Perkin, S. The electrostatic screening length in concentrated electrolytes increases with concentration. J. Phys. Chem. Lett. 2016, 7, 2157-2163.

(10) Atkin, R.; El Abedin, S. Z.; Hayes, R.; Gasparotto, L. H. S.; Borisenko, N.; Endres, F. AFM and STM studies on the surface interaction of [BMP]TFSA and [EMIm]TFSA ionic liquids with $\mathrm{Au}(111)$. J. Phys. Chem. C 2009, 113, 13266-13272.

(11) Wallauer, J.; Drüschler, M.; Huber, B.; Roling, B. The Differential Capacitance of Ionic Liquid / Metal Electrode Interfaces - A Critical Comparison of Experimental Results with Theoretical Predictions. Z. Naturforsch., B: J. Chem. Sci. 2013, 68, 1143-1153.

(12) Alam, M. T.; Islam, M. M.; Okajima, T.; Ohsaka, T. Capacitance measurements in a series of room-temperature ionic liquids at glassy carbon and gold electrode interfaces. J. Phys. Chem. C 2008, 112, 16600-16608.

(13) Costa, R.; Pereira, C. M.; Silva, F. Double layer in room temperature ionic liquids: influence of temperature and ionic size on the differential capacitance and electrocapillary curves. Phys. Chem. Chem. Phys. 2010, 12, 11125-11132.

(14) Fedorov, M. V.; Kornyshev, A. A. Towards understanding the structure and capacitance of electrical double layer in ionic liquids. Electrochim. Acta 2008, 53, 6835-6840.

(15) Atkin, R.; Borisenko, N.; Drüschler, M.; Endres, F.; Hayes, R.; Huber, B.; Roling, B. Structure and dynamics of the interfacial layer between ionic liquids and electrode materials. J. Mol. Liq. 2014, 192, 44-54.

(16) Hoffmann, V.; Pulletikurthi, G.; Carstens, T.; Lahiri, A.; Borodin, A.; Schammer, M.; Horstmann, B.; Latz, A.; Endres, F. Influence of a silver salt on the nanostructure of a $\mathrm{Au}(111) /$ ionic liquid interface: an atomic force microscopy study and theoretical concepts. Phys. Chem. Chem. Phys. 2018, 20, 4760-4771.

(17) Borisenko, N.; Zein El Abedin, S.; Endres, F. In situ STM investigation of gold reconstruction and of silicon electrodeposition on $\mathrm{Au}(111)$ in the room temperature ionic liquid 1-butyl-1methylpyrrolidinium bis(trifluoromethylsulfonyl)imide. J. Phys. Chem. B 2006, 110, 6250-6256.

(18) Uhl, B.; Cremer, T.; Roos, M.; Maier, F.; Steinrück, H.-P.; Behm, R. J. At the ionic liquidlmetal interface: structure formation and temperature dependent behavior of an ionic liquid adlayer on $\mathrm{Au}(111)$. Phys. Chem. Chem. Phys. 2013, 15, 17295-17302.

(19) Wen, R.; Rahn, B.; Magnussen, O. M. Potential-dependent adlayer structure and dynamics at the ionic liquid/ $\mathrm{Au}(111)$ interface: A molecular-scale in situ video-STM study. Angew. Chem., Int. Ed. 2015, 54, 6062-6066.

(20) Wen, R.; Rahn, B.; Magnussen, O. M. In situ video-STM study of adlayer structure and surface dynamics at the ionic liquid/Au (111) Interface. J. Phys. Chem. C 2016, 120, 15765-15771.

(21) Buchner, F.; Forster-Tonigold, K.; Uhl, B.; Alwast, D.; Wagner, N.; Farkhondeh, H.; Gross, A.; Behm, R. J. Toward the microscopic identification of anions and cations at the ionic $\operatorname{liquid} \operatorname{Ag}(111)$ interface: a combined experimental and theoretical investigation. ACS Nano 2013, 7, 7773-7784.

(22) Su, Y.-Z.; Yan, J.-W.; Li, M.-G.; Xie, Z.-X.; Mao, B.-W.; Tian, Z.-Q. Adsorption of solvent cations on $\mathrm{Au}(111)$ and $\mathrm{Au}(100)$ in alkylimidazolium-based ionic liquids - worm-like versus micelle-like structures. Z. Phys. Chem. 2012, 226, 979-994.

(23) Limmer, D. T. Interfacial ordering and accompanying divergent capacitance at ionic liquid-metal interfaces. Phys. Rev. Lett. 2015, 115, 256102.

(24) Motobayashi, K.; Minami, K.; Nishi, N.; Sakka, T.; Osawa, M. Hysteresis of potential-dependent changes in ion density and structure of an ionic liquid on a gold electrode: In situ observation by surface-enhanced infrared absorption spectroscopy. J. Phys. Chem. Lett. 2013, 4, 3110-3114.

(25) Motobayashi, K.; Nishi, N.; Inoue, Y.; Minami, K.; Sakka, T.; Osawa, M. Potential-induced restructuring dynamics of ionic liquids on a gold electrode: Steric effect of constituent ions studied by surface-enhanced infrared absorption spectroscopy. J. Electroanal. Chem. 2017, 800, 126-133.

(26) Lauw, Y.; Horne, M. D.; Rodopoulos, T.; Lockett, V.; Akgun, B.; Hamilton, W. A.; Nelson, A. R. J. Structure of [C4mpyr][NTf2] Room-Temperature Ionic Liquid at Charged Gold Interfaces. Langmuir 2012, 28, 7374-7381.

(27) Lockett, V.; Horne, M.; Sedev, R.; Rodopoulos, T.; Ralston, J. Differential capacitance of the double layer at the electrode/ionic liquids interface. Phys. Chem. Chem. Phys. 2010, 12, 12499-12512.

(28) Sebastián, P.; Sandoval, A. P.; Climent, V.; Feliu, J. M. Study of the interface $\mathrm{Pt}(111) /[\mathrm{Emmim}][\mathrm{NTf} 2]$ using laser-induced temperature jump experiments. Electrochem. Commun. 2015, 55, 39-42.

(29) Sebastián, P.; Climent, V.; Feliu, J. M. Characterization of the interfaces between $\mathrm{Au}(\mathrm{hkl})$ single crystal basal plane electrodes and [Emmim] [Tf $2 \mathrm{~N}]$ ionic liquid. Electrochem. Commun. 2016, 62, 4447.

(30) Frittmann, S.; Halka, V.; Jaramillo, C.; Schuster, R. An improved sensor for electrochemical microcalorimetry, based on lithiumtantalate. Rev. Sci. Instrum. 2015, 86, 064102.

(31) Frittmann, S.; Halka, V.; Schuster, R. Identification of NonFaradaic Processes by Measurement of the Electrochemical Peltier Heat during the Silver Underpotential Deposition on $\mathrm{Au}(111)$. Angew. Chem., Int. Ed. 2016, 55, 4688-4691.

(32) Agar, J. N. Thermogalvanic Cells. In Advances in Electrochemistry and Electrochemical Engineering; Delahay, P., Ed.; Interscience Publishers: London, 1963; Vol. 3, pp 31-121.

(33) Ozeki, T.; Ogawa, N.; Aikawa, K.; Watanabe, I.; Ikeda, S. Thermal analysis of electrochemical reactions: Influence of electrolytes on peltier heat for $\mathrm{Cu}(0) / \mathrm{Cu}(\mathrm{II})$ and $\mathrm{Ag}(0) / \mathrm{Ag}(\mathrm{I})$ redox systems. J. Electroanal. Chem. 1983, 145, 53-65.

(34) Schuster, R. Electrochemical microcalorimetry at single electrodes. Curr. Opin. Electrochem. 2017, 1, 88-94.

(35) Etzel, K. D.; Bickel, K. R.; Schuster, R. A microcalorimeter for measuring heat effects of electrochemical reactions with submonolayer conversions. Rev. Sci. Instrum. 2010, 81, 034101.

(36) Frittmann, S.; Schuster, R. Role of anions during the $\mathrm{Cu}$ underpotential deposition on $\mathrm{Au}(111)$ : A microcalorimetric investigation. J. Phys. Chem. C 2016, 120, 21522-21535.

(37) Bickel, K. R.; Etzel, K. D.; Halka, V.; Schuster, R. Microcalorimetric determination of heat changes caused by overpotential upon electrochemical Ag bulk deposition. Electrochim. Acta 2013, 112, 801-812.

(38) Bickel, K. R.; Timm, A. E.; Nattland, D.; Schuster, R. Microcalorimetric determination of the entropy change upon the electrochemically driven surface aggregation of dodecyl sulfate. Langmuir 2014, 30, 9085-9090.

(39) MacFarlane, D. R.; Meakin, P.; Sun, J.; Amini, N.; Forsyth, M. Pyrrolidinium imides: A new family of molten salts and conductive plastic crystal phases. J. Phys. Chem. B 1999, 103, 4164-4170.

(40) Alwast, D.; Schnaidt, J.; Law, Y. T.; Behm, R. J. A novel approach for differential electrochemical mass spectrometry studies 
on the decomposition of ionic liquids. Electrochim. Acta 2016, 197, 290-299.

(41) Zhong, Y.; Yan, J.; Li, M.; Chen, L.; Mao, B. The electric double layer in an ionic liquid incorporated with water molecules: atomic force microscopy force curve study. ChemElectroChem 2016, 3, $2221-2226$.

(42) Uhl, B.; Buchner, F.; Alwast, D.; Wagner, N.; Behm, R. J. Adsorption of the ionic liquid [BMP][TFSA] on $\mathrm{Au}(111)$ and $\mathrm{Ag}(111)$ : substrate effects on the structure formation investigated by STM. Beilstein J. Nanotechnol. 2013, 4, 903-918.

(43) Friedl, J.; Markovits, I. I. E.; Herpich, M.; Feng, G.; Kornyshev, A. A.; Stimming, U. Interface between an $\mathrm{Au}(111)$ surface and an ionic liquid: The influence of water on the double-layer capacitance. ChemElectroChem 2017, 4, 216-220.

(44) Pajkossy, T.; Müller, C.; Jacob, T. The metal-ionic liquid interface as characterized by impedance spectroscopy and in situ scanning tunneling microscopy. Phys. Chem. Chem. Phys. 2018, 20, 21241-21250.

(45) Preiss, U.; Bulut, S.; Krossing, I. In Silico Prediction of the Melting Points of Ionic Liquids from Thermodynamic Considerations: A Case Study on 67 Salts with a Melting Point Range of 337 ${ }^{\circ}$ C. J. Phys. Chem. B 2010, 114, 11133-11140.

(46) Fedorov, M. V.; Georgi, N.; Kornyshev, A. A. Double layer in ionic liquids: The nature of the camel shape of capacitance. Electrochem. Commun. 2010, 12, 296-299.

(47) Lindner, J. Elektrochemische und mikrokalorimetrische Untersuchung des zeit- und potentialabhängigen Doppelschichtverhaltens von Ionischen Flüssigkeiten an Gold(111). Dissertation, KIT: Karlsruhe, 2019.

(48) Koepp, H.-M.; Wendt, H.; Strehlow, H. Der Vergleich der Spannungsreihen in verschiedenen Solventien. II. Z. Elektrochem. 1960, 64, 483-491.

(49) Schlag, K.; Braun, J.; Nattland, D.; Schuster, R. Unraveling compositional changes of the double layer upon sulfate adsorption on $\mathrm{Au}(111)$ by surface plasmon resonance. J. Phys. Chem. C 2019, 123, 24598.

(50) Boudeville, P. Thermometric determination of electrochemical Peltier heat (thermal effect associated with electron transfer) of some redox couples. Inorg. Chim. Acta 1994, 226, 69-78.

(51) Shi, Z.; Lipkowski, J.; Gamboa, M.; Zelenay, P.; Wieckowski, A. Investigations of $\mathrm{SO} 42-$ adsorption at the $\mathrm{Au}(111)$ electrode by chronocoulometry and radiochemistry. J. Electroanal. Chem. 1994, $366,317-326$.

(52) Drüschler, M.; Borisenko, N.; Wallauer, J.; Winter, C.; Huber, B.; Endres, F.; Roling, B. New insights into the interface between a single-crystalline metal electrode and an extremely pure ionic liquid: slow interfacial processes and the influence of temperature on interfacial dynamics. Phys. Chem. Chem. Phys. 2012, 14, 5090-5099.

(53) Roling, B.; Drüschler, M.; Huber, B. Slow and fast capacitive process taking place at the ionic liquid/electrode interface. Faraday Discuss. 2012, 154, 303-311.

(54) Vargas-Barbosa, N. M.; Roling, B. Time-resolved determination of the potential of zero charge at polycrystalline $\mathrm{Au}$ /ionic liquid interfaces. J. Chem. Phys. 2018, 148, 193820.

(55) Pitzer, K. S. Thermodynamics of thermocells with fused or solid electrolytes. J. Phys. Chem. 1961, 65, 147-150.

(56) Krossing, I.; Slattery, J. M.; Daguenet, C.; Dyson, P. J.; Oleinikova, A.; Weingärtner, H. Why are ionic liquids liquid? A simple explanation based on lattice and solvation energies. J. Am. Chem. Soc. 2006, 128, 13427-13434.

(57) Tokuda, H.; Ishii, K.; Susan, M. A. B. H.; Tsuzuki, S.; Hayamizu, K.; Watanabe, M. Physicochemical properties and structures of room-temperature ionic liquids. 3. Variation of cationic structures. J. Phys. Chem. B 2006, 110, 2833-2839.

(58) Harrison, J.; Randles, J. E. B.; Schiffrin, D. J. The entropy of formation of the mercury-aqueous solution interface and the structure of the inner layer. J. Electroanal. Chem. 1973, 48, 359-381.

(59) Garcia-Araez, N.; Climent, V.; Feliu, J. M. Analysis of temperature effects on hydrogen and $\mathrm{OH}$ adsorption on $\mathrm{Pt}(111)$,
$\mathrm{Pt}(100)$ and $\mathrm{Pt}(110)$ by means of Gibbs thermodynamics. J. Electroanal. Chem. 2010, 649, 69-82.

(60) Guidelli, R.; Aloisi, G.; Leiva, E.; Schmickler, W. Contribution to the entropy of formation of metal/solution interphases from metal electrons. J. Phys. Chem. 1988, 92, 6671-6675.

(61) Silva, F.; Gomes, C.; Figueiredo, M.; Costa, R.; Martins, A.; Pereira, C. M. The electrical double layer at the [BMIM][PF6] ionic liquid/electrode interface - Effect of temperature on the differential capacitance. J. Electroanal. Chem. 2008, 622, 153-160.

(62) Lockett, V.; Sedev, R.; Ralston, J.; Horne, M.; Rodopoulos, T. Differential capacitance of the electrical double layer in imidazoliumbased ionic liquids: Influence of potential, cation Size, and temperature. J. Phys. Chem. C 2008, 112, 7486-7495.

(63) Hill, T. L. An introduction to statistical thermodynamics, 1st ed.; Dover Publications: Onatrio, 1987.

(64) Downing, R.; Bossa, G. V.; May, S. The Role of Ion-Ion Correlations for the Differential Capacitance of Ionic Liquids. J. Phys. Chem. C 2018, 122, 28537-28544.

(65) Kornyshev, A. A.; Qiao, R. Three-dimensional double layers. J. Phys. Chem. C 2014, 118, 18285-18290.

(66) Lahiri, A.; Carstens, T.; Atkin, R.; Borisenko, N.; Endres, F. In situ atomic force microscopic studies of the interfacial multilayer nanostructure of LiTFSI-[Py 1, 4]TFSI on $\mathrm{Au}(111)$ : Influence of $\mathrm{Li}$ + ion concentration on the $\mathrm{Au}(111) / \mathrm{IL}$ interface. J. Phys. Chem. C 2015, 119, 16734-16742.

(67) Georgi, N.; Kornyshev, A. A.; Fedorov, M. V. The anatomy of the double layer and capacitance in ionic liquids with anisotropic ions: Electrostriction vs. lattice saturation. J. Electroanal. Chem. 2010, 649, 261-267. 